\title{
Measurement Method and Application of a Deep Learning Digital Economy Scale Based on a Big Data Cloud Platform
}

Yanmei Zhao, Northeast Normal University, Changchun, China \& ChangChun Finance College, Changchun, China

* Yixin Zhou, Shanghai Business School, Shanghai, China

\begin{abstract}
In recent years, with the acceleration of the process of economic globalization and the deepening of China's financial liberalization, the scale of international short-term capital flows has been extremely rapid. This article mainly studies the deep learning digital economy scale measurement method and its application based on the big data cloud platform. This article uses the indirect method to estimate the stock of renminbi circulating abroad. The results show that the application of big data cloud platforms can increase the development share of digital media and digital transactions in the digital economy and optimize the structure of China's digital economy.
\end{abstract}

\section{KEYWORDS}

Big Data, Cloud Platform, Deep Learning, Digital Economy, Scale Measurement Method

\section{INTRODUCTION}

The digital economy industry has become the most representative high-tech industry, deeply integrated with other industries, plays a significant role in improving industrial efficiency and promoting new industries, and has become a new driving force for China's economic transformation and upgrading. Countries around the world have regarded the development of digital economy as an important part of economic growth and improving international competitiveness (Kitouni 2018).

Today's world has entered an important period in which information technology, represented by the Internet, has penetrated into the field of traditional economy and promoted the transformation of various economies to digital economy. The innovation of business model and the change of economic format in digital economy have greatly reduced the economic cost and greatly improved the efficiency of International trade, which has a significant impact on the division of global value chain. With the enhancement of China's economic strength, the deepening of financial reform and the improvement of the market (Yeh nd), RMB will not only increase greatly in the scale of overseas circulation, but also continuously enrich its functions, and gradually transition from sovereign currency to international currency.

With the development of computer technology, all kinds of scientific industries have achieved unprecedented development. Long $\mathrm{M}$ thinks that domain adaptation generalizes learning models across source and target domains, which are sampled from different distributions. In order to enhance the invariance of depth representation and make it more transferable in various fields, he proposed 
a unified depth adaptation framework for joint learning of transitive representation and classifier, so as to make full use of the advantages of depth learning and optimal two sample matching to achieve scalable domain adaptation. The framework includes two interdependent paradigms, namely, unsupervised pre training using depth de-noising automatic encoder for effective training of depth model, and supervised fine-tuning using depth neural network for effective utilization of discrimination information. Both of them learn by embedding depth representation into regenerative kernel. Although his research is tenable in theory, there is no concrete experiment to prove it (Long 2016). Charalampous K proposed an unsupervised online deep learning algorithm for video sequence action recognition. Each computing node of his example forms a cluster and calculates the point representation (Lv 2020). Then, the first-order transformation matrix stores and continuously updates the continuous transformations between clusters. Viterbi algorithm deals with both spatial and temporal information. Although the algorithm he used has good performance, there are errors in the calculation results (Charalampous 2016). Zhang L believes that in the past few decades, people have developed machine learning tools, such as quantitative structure-activity relationship (QSAR) modeling, which can quickly and cheaply identify potential bioactive molecules from millions of candidate compounds. He summarized the history of machine learning and provided insights on the recently developed deep learning methods and their applications in rational drug discovery. Although his research is more comprehensive, many definitions are not accurate (Zhang 2017). Mahmud M believes that the improvement of computing power, along with the increase of data storage speed and the decrease of computing cost, has enabled scientists in various fields to apply these technologies to data sets that were difficult to process due to scale and complexity. He will conduct a comprehensive investigation on the application of DL, RL and deep RL technology in biological data mining (Feng 2020). In addition, he compared the performance of applying DL technology to different datasets in various application domains. Finally, he outlined the problems to be solved in this challenging research area and discussed the future development prospects. Although his research is comprehensive, there is a lack of experimental data (Mahmud 2017).

Based on the current mainstream economic theory, this paper makes a further analysis on the mechanism and ways of the impact of digital economy development on export trade. At the same time, the digital economy continues to spawn new industries and new formats, which will also have a direct or indirect impact on export trade. This paper analyzes the change of added value of value chain links in the process of value chain decomposition, integration, innovation and reconstruction from three levels of digital economy to micro enterprise value chain, meso traditional industry chain and macro global value chain, and then puts forward the theoretical mechanism of the influence of digital economy on China's division of labor in global value chain.

\section{MEASUREMENT OF THE SCALE OF THE DEEP LEARNING DIGITAL ECONOMY}

\subsection{Big Data Cloud Platform}

An open source, scalable, and distributed infrastructure for data processing (Z. Lv and B. Hu 2020). Hadoop can be deployed on one to several thousands of ordinary computer nodes, use distributed file system to provide storage of large amounts of data, and use parallel programming model to process and analyze large amounts of data stored in distributed file system. Each node in the Hadoop cluster provides local storage and local computing, and the local storage and local computing of all nodes are uniformly organized to form a larger and more efficient storage and computing cluster (Hao 2016).

The architecture of Nova's management virtual machine is shown in Figure 1. The API is the only way for the client to access Nova. The API accepts the requests passed by the client to Nova, converts these requests into AMQP messages and puts them in the Queue, and then returns the obtained data results to the client after Nova has processed them. It is precisely based on the above mechanism 


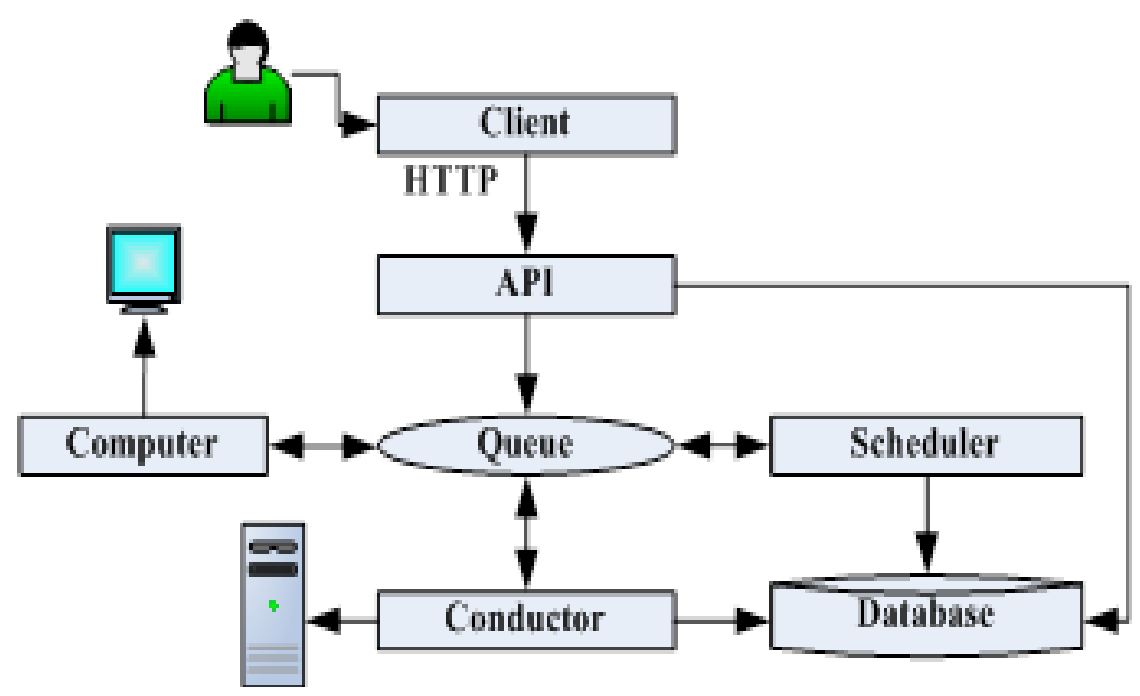

that the API needs to have a high degree of stability, and any modification needs to be explained by a special bp, otherwise it will not be approved. In the process of creating a virtual machine, the API receives the client's creation request, invokes the Conductor service through the Queue, the Conductor prepares the relevant parameters of the virtual machine, and then calls the Scheduler service through the Queue to select the appropriate physical node for the virtual machine, and the Conductor obtains the target host. After the information, call the Compute service to create a new virtual machine (He 2018; Asaoka 2016).

The resource manager plays the role of manager in the whole architecture. It usually allocates a single computer to run in the way of back-end service process. When the resource scheduling of each program can be allocated by the resource manager. In order to achieve resource scheduling, resource manager will save the information of idle resources and nodes in the cluster, and guide users to obtain the required content according to their own standard shared allocation decision algorithm and stored information when users initiate requests (W Wang 2016; C Wang2017; Yang 2020).

In the data interaction function part, it is responsible for the data interaction function between the upper data warehouse part of the system and the external data sources, which allows the data of external data to be imported into the data warehouse for persistent storage, and also supports the data in the data warehouse to be exported to the external storage system when permission permits. Through its memory storage characteristics, it provides a cache engine for high-speed reading data in data exchange. At the same time, the socket programming method is adopted to realize the function of reading redis data information, and the sqoop tool is integrated to realize the exchange between data warehouse and data source in a distributed way (Koesdwiady 2016).

In these resources, consumers can deploy different operating systems and different applications. When an enterprise constructs a project, it needs to purchase and deploy servers, arrange operation and maintenance personnel to maintain servers, so as to ensure the safe use of data. When there is a large amount of data, it also needs to clean up the previous data, make room to store new data, or purchase new servers (Zhang 2017). As a whole, hardware equipment and operation and maintenance personnel need high expenses (Zeng 2016). 


\subsection{Deep Learning}

In terms of the computational complexity of the network structure, if a network with a depth of $\mathrm{n}$ can exactly express a certain high-order objective function, then a network model less than $\mathrm{n}$ should be used to achieve the same effect. Since the calculation factor increases exponentially, the calculation The amount will increase greatly, and the learning effect of the network is not necessarily very good. The idea of automatic encoding is to map the original data from one representation space to another spatial representation, and then map it back from the new spatial representation back to the original feature space, and update the network with the goal of minimizing the reconstruction error. The parameters make the reconstructed data and the original data as close as possible (Zhao 2017).

The GM(1,1) model can be expressed as the following form:

$$
\frac{d s^{(1)}}{d t}+a s^{(1)}=b
$$

$$
s^{(1)}(k+1)=\left[s^{(0)}(1)-\frac{b}{a}\right] e^{-a k}+\frac{b}{a}
$$

Among them, $s^{(1)}(k)$ is called the cumulative generating sequence (J Wang 2017; Ghesu 2016). Generally, the calculation formula of the model parameter column $\hat{a}$ in the $\operatorname{GM}(1,1)$ model is as follows:

$$
\hat{a}=\left[\begin{array}{l}
a \\
b
\end{array}\right]=\left(B^{T} B\right)^{-1} B^{T} Y
$$

$$
\begin{aligned}
& Y=\left[\begin{array}{c}
s^{(0)}(2) \\
s^{(0)}(3) \\
\vdots \\
s^{(0)}(n)
\end{array}\right] \\
& B=\left[\begin{array}{cc}
-z^{(1)}(2) & 1 \\
-z^{(1)}(3) & 1 \\
\vdots & \vdots \\
-z^{(1)}(n) & 1
\end{array}\right]
\end{aligned}
$$

The characteristics of each sample in the training set can be regarded as the description of the site, and the label can be regarded as the correct action to be performed. Unfortunately, supervised learning can't learn to interact smoothly, because it is very impractical to get data and labels with expected behavior in an interactive environment. The system can only be trained from its own experience, so the behavior in experience must be the best (Li 2016; Edara 2020). 
In order to achieve better training and learning effect of DBN, the learning process mainly includes layer by layer independent pre training and error feedback tuning from low level to high level. Because the input of each layer is the output of the upper layer, which has no direct relationship with the higher layer, it can reduce the complexity of the traditional deep neural network, avoid the loss of the error return of the traditional deep neural network due to multiple layers, and improve the recognition effect (Chen 2017).

In training, a feature pool for storing eigenvalues and a classifier pool for representing classifiers are established, and then a feature subset and a corresponding classifier are selected for each category. In testing, several classifiers are used for each test sample to obtain their own classification results, and then the final classification results are obtained by synthesizing multiple classifiers (Biswas 2018). Because LDA is a supervised dimension reduction algorithm, in most cases, the features reduced by LDA are more effective for classification than those reduced by PCA (X Wang 2016; Albarqouni 2016).

\subsection{Methods for Measuring the Scale of Digital Economy}

Behind the development of digital economy is the continuous evolution of digital technology, and digital technology itself maintains its own life through continuous innovation. In other words, the rapid development of digital technology determines that digital economy is not an unchangeable economic form, and its industry scope and characteristics are constantly enriched and changed with the development of the times (W Zhao 2016). In the process of modeling, the time series corresponding to the system behavior dependent variable $S_{l}$ is expressed as follows:

$$
S_{l}^{(0)}=\left\{s_{l}^{(0)}(k)\right\}=\left\{s_{l}^{(0)}(1), s_{l}^{(0)}(2), \ldots, s_{l}^{(0)}(k), \ldots, s_{l}^{(0)}(n)\right\}
$$

The sequence corresponding to the system behavior argument $F_{1}, F_{2}, \ldots, F_{M}$ is expressed as follows:

$$
F_{m}^{(0)}=\left\{f_{m}^{(0)}(k)\right\}=\left\{f_{m}^{(0)}(1), f_{m}^{(0)}(2), \ldots, f_{m}^{(0)}(k), \ldots, f_{m}^{(0)}(n)\right\}
$$

The formula for calculating the error of the model-based training method is shown in the following formula:

$$
E=\frac{l}{2}(D-O)^{2}=\frac{l}{2} \sum_{k=1}^{i}\left(d_{k}-o_{k}\right)^{2}
$$

Among them, $\mathrm{O}$ is the actual output and $\mathrm{D}$ is the expected output (Marmanis 2016; Zhu 2018). The calculation error formula for the training method based on batch and block update is as follows:

$$
E_{\min }=\frac{1}{2} \sum_{p=1}^{p} \sum_{k=1}^{i}\left(d_{k}^{p}-o_{k}^{p}\right)^{2}
$$

Among them, $\mathrm{D}$ is the expected output, $\mathrm{O}$ is the actual output, and $\mathrm{P}$ is the total number of samples (Kallenberg 2016; Nguyen 2016).

The feature mapping function $h^{k}$ can be expressed as: 


$$
h_{i j}^{k}=f\left(\sum_{i \in M_{j}} X_{i}^{k-1} * W_{i j}^{k}+b^{k}\right)
$$

The energy function of RBM is defined as:

$$
E(v, h)=-\sum_{i=1}^{n} \sum_{j=1}^{m} w_{i j} h_{i} v_{j}-\sum_{j=1}^{m} b_{j} v_{j}-\sum_{i=1}^{n} b_{i} c_{i}
$$

When the RBM layer reaches stability, the probability of occurrence of a certain input vector is only determined by its energy and the energy of all possible state vectors:

$$
p(v)=\frac{e^{-E(v)}}{\sum_{t} e^{-E(t)}}
$$

The appropriate scale of farmland management is a dynamic and relative concept. Different regions or different development stages of the same region have different conditions such as land resource abundance, agricultural production and management entities, agricultural production technology and equipment, and social and economic development conditions. There will be differences in the appropriate scale of farmland management. The comprehensive evaluation of farmland scale management conditions requires that the distribution characteristics of the projection value Z(i) are small concentration and large dispersion. Therefore, the projection indicator function is:

$Q(a)=S_{z} D_{z}$

$S_{z}=\sqrt{\frac{\sum_{i=1}^{n}(z(j)-E(z))^{2}}{n-1}}$

$D_{z}=\sum_{i=1}^{n} \sum_{j=1}^{n}(R-r(i, j)) u(R-r(i, j))$

In the formula, $\mathrm{E}(\mathrm{z})$ is the mean value of the projection value sequence, and $\mathrm{R}$ is the window radius of the local density (Yan 2016).

Due to the rapid development of China's real estate market and stock market, as well as the further opening of China's finance and economy, and the stock market and land market absorb a large amount of funds from China's market, stock price and house price also have a certain impact on money demand. The intermediate products provided by the countries with digital economy advantages can not only meet the needs of their own development, but also meet the price demands of the countries with digital economy disadvantages for the realization of enterprise profits (Hosseini-Asl 2016). 


\section{DEEP LEARNING DIGITAL ECONOMY SCALE MEASUREMENT EXPERIMENT}

\subsection{Data Sources}

The annual interest rate of China's three-month time deposit is from the website of the People's Bank of China, and the market annual interest rate of the large negotiable certificate of deposit with maturity of three months in the United States is from the website of the Federal Reserve board. The RMB exchange rate adopts the central parity of the RMB exchange rate against the US dollar published on the website of the People's Bank of China. The rate of return on the capital market is calculated using the quarterly closing price of the Shanghai Stock Exchange, which is derived from Flush Software. The quarterly GDP data comes from the website of the national bureau of Statistics of China. Since the influencing factors of capital flight usually have different manifestations in different periods and in different countries and regions, considering the actual situation in China and the requirements of the measurement model for sample size, this paper selects the expected depreciation of the domestic currency, the actual domestic and foreign interest rate differentials, and the foreign debt situation. Empirical analysis of foreign direct investment as a possible influencing factor of capital flight.

\subsection{Data Processing}

This paper takes the natural logarithm of M0t/CPIt, GDPt/CPIt, St, and Ht to reduce the heteroscedasticity of the model. The one-year time deposit interest rate $\mathrm{R}$ is a weighted average of the quarterly interest rate based on the time of interest rate adjustment announced by the central bank. The software used in this article is Eviews5.0. Because it is a quarterly data, some data may have seasonal changes, and seasonal changes will obscure the law of economic development. Therefore, when we use these data, we make seasonal adjustments to the data with seasonal factors.

\subsection{Construction of the Scale Calculation Model}

This article uses the indirect method to estimate the stock of renminbi circulating abroad. Under the circumstance that the renminbi does not flow out, the total currency supply of a country should be exactly equal to its domestic demand; then, in the case of renminbi outflow, the analysis is carried out in stages.

\subsection{Stationarity Test}

The dependent and independent variables in this article are both time series. Before establishing the model, the stationarity of the time series must be unit and tested. The stationary series have a mean reversion trend, and the volatility around its mean is approximately constant, so the model prediction is reliable of. The ADF unit and test method is adopted, and the determination of the lagging term adopts the SIC criterion.

\subsection{Cointegration Test}

Johanson cointegration test is to calculate the trace statistics and the largest eigenvalue statistics to make judgments. The inspection method of the trace statistics is more special, and the circular inspection method is adopted (H Chen 2020).

\subsection{Unit Testing}

This article uses the TUT framework to unit test the system. TUT is a lightweight framework written using template technology. There are only two header files in its code. In use, you only need to copy these two header files to the project directory or set the include path of the header file when writing the unit test. There is no need to set up complicated compilation links. The first step in the process of conducting big data experiments on this platform for multiple users is to apply for a cluster to deploy a big data experiment environment. Deployment time can greatly affect user experience. 
Table 1. Results of ADF inspection method

\begin{tabular}{|l|l|l|l|l|}
\hline Variable & $\begin{array}{l}\text { Situation }(\mathbf{C}, \\
\text { T, I })\end{array}$ & $\begin{array}{l}\text { ADF inspection } \\
\text { value }\end{array}$ & Critical value & $\begin{array}{l}\text { Probability } \\
\text { value }\end{array}$ \\
\hline SCF & $(0,0,0)$ & -4.039799 & $\begin{array}{l}(-3.626784,-2.945842,- \\
2.611531)\end{array}$ & 0.0034 \\
\hline R & $(0,0,0)$ & -3.061233 & $\begin{array}{l}(-3.626784,-2.945842,- \\
2.611531)\end{array}$ & 0.0387 \\
\hline E & $(0,0,1)$ & -2.736048 & $\begin{array}{l}(-3.632900,-2.948404,- \\
2.612874)\end{array}$ & 0.0782 \\
\hline SR & $(0,0,3)$ & -3.762465 & $\begin{array}{l}(-3.646342,-2.954021,- \\
2.615817)\end{array}$ & 0.0075 \\
\hline GGDP & $(0,0,4)$ & -3.107894 & $\begin{array}{l}(-3.653730,-2.957110,- \\
2.617434)\end{array}$ & 0.0360 \\
\hline
\end{tabular}

\section{RESULTS AND DISCUSSION}

\subsection{Measurement and Analysis of the Scale of the Digital Economy}

The results of the ADF test method are shown in Table 1. From the data in the table, it can be seen that the short-term cross-border capital SCF, the capital market rate of return SR, and the economic growth rate GGDP are stable time series at the significance level of $1 \%$, and the change in the SinoUS interest rate difference $\mathrm{R}$ is at the significance level of 5\%. The above is a stable time series, and the RMB exchange rate change $\mathrm{E}$ is a stable time series at the significance level of $10 \%$ (Wen 2021). Strictly speaking, in a multivariate VAR model, all variables should be jointly stable. The variables in this paper all meet the basic prerequisites for entering the VAR model.

The performance comparison is shown in Figure 2. In all test cases, the hybrid architecture of GlusterFS and MapReduce performs faster than the separate architecture for the same task. This is because in the hybrid architecture, the computing node and the storage node are in the same cluster, and the data access efficiency can be improved by the principle of data proximity.

The decomposition of economic growth in different periods is shown in Table 2. In the whole period, the contribution of non ICT capital to economic growth is the largest, with an average contribution of $53.08 \%$. In addition, the contribution of total factor productivity ranks second, with

Figure 2. Performance comparison

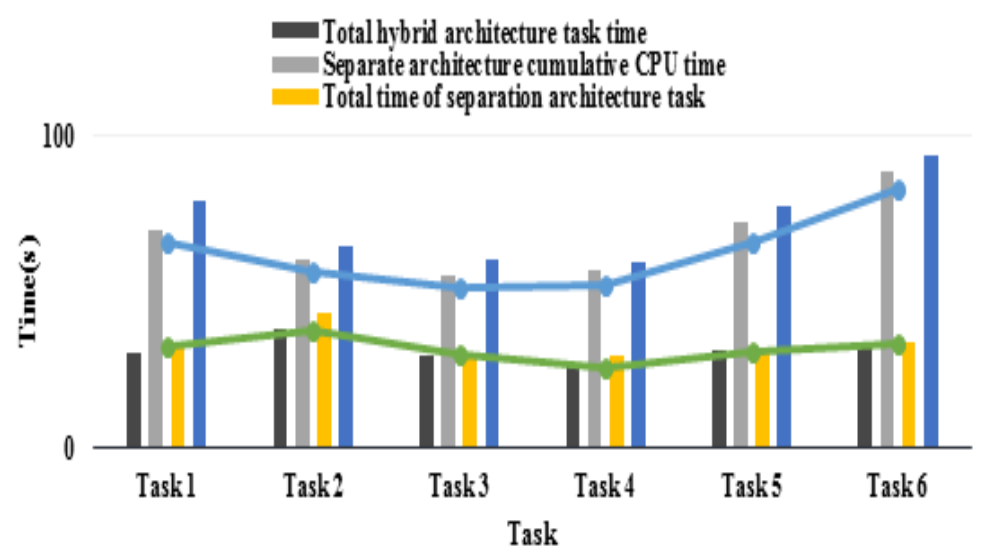


Table 2. Breakdown of economic growth by periods

\begin{tabular}{|l|l|l|l|}
\hline ICT capital contribution & Non-ICT capital contribution & Labor contribution & TFP contribution \\
\hline $6.21 \%$ & $53.08 \%$ & $4 \%$ & $36.67 \%$ \\
\hline $4.12 \%$ & $50.07 \%$ & $5.40 \%$ & $40.36 \%$ \\
\hline $8.10 \%$ & $45.47 \%$ & $4.70 \%$ & $42.20 \%$ \\
\hline $4.43 \%$ & $59.11 \%$ & $2.10 \%$ & $34.36 \%$ \\
\hline $7.60 \%$ & $66.45 \%$ & $2.60 \%$ & $23.35 \%$ \\
\hline
\end{tabular}

an average contribution of $36.67 \%$, while the contribution of ICT capital is slightly higher than that of labor, with $6.21 \%$ and $4 \%$ respectively. From 1992 to 1999 , the contribution of ICT capital was only $4.12 \%$, while the contribution of labor was $5.4 \%$, which was higher than that of ICT capital. The contribution rates of non ICT capital and TFP were $50.07 \%$ and $40.36 \%$. From the error correction term, in the long-term equilibrium, there is a positive correlation between the scale of China's digital economy LDE and the scale of software industry LSOFT. According to the cointegration equation, the elasticity of the scale of China's software industry to the growth of the scale of digital economy is 0.78 ( $\mathrm{Z} \mathrm{Lv}$ and N Kumar 2020), which indicates that the logarithmic value of the scale of digital economy increases by $0.78 \%$ with every $1 \%$ increase in the logarithmic value of the scale of software industry under other conditions. Therefore, in the long run, the development of software industry will promote the growth of digital economy.

The result of native deployment is shown in Figure 3. The experiment recorded the longest deployment time of the cluster when the number of users was $1,5,10,20$, and 30, respectively. The test is performed five times in total, and the average value is taken. Among them, redeploy the clusters that were not successfully deployed for the first time. When five or less users deploy clusters at the same time, OpenStack can smoothly process tasks. As the number of users increases, the CPU load on the node will gradually increase, which will cause the communication between the control node and the computing node to stagnate, and the cluster creation fails (Shankar 2019). The deployment time for about 20 users has been long, and failures have increased. The one-click deployment scenario uses a cluster pool mechanism to achieve second-level deployment when the number of users does not exceed the capacity of the cluster pool. When the number of users exceeds the capacity of the cluster pool, the response time of some users has increased, but the number of successes is still

Figure 3. Native deployment result

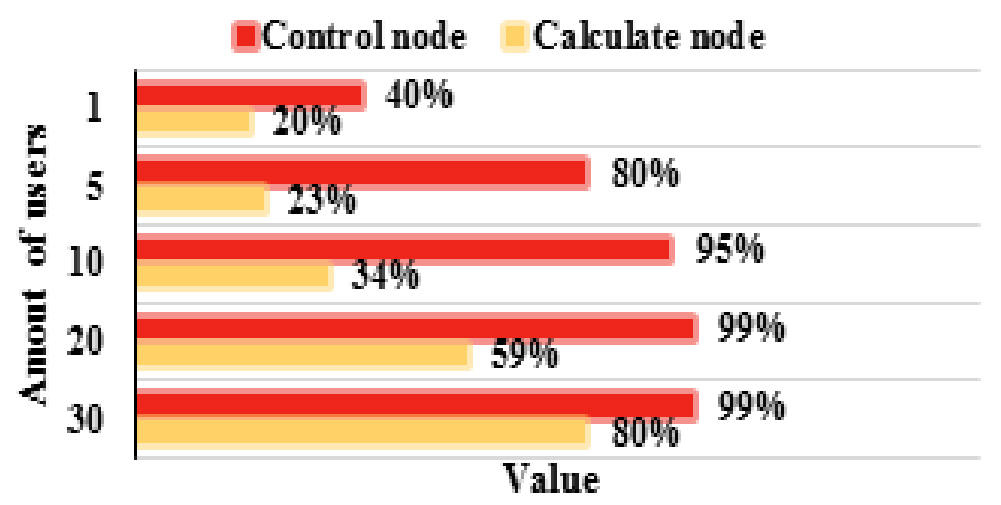




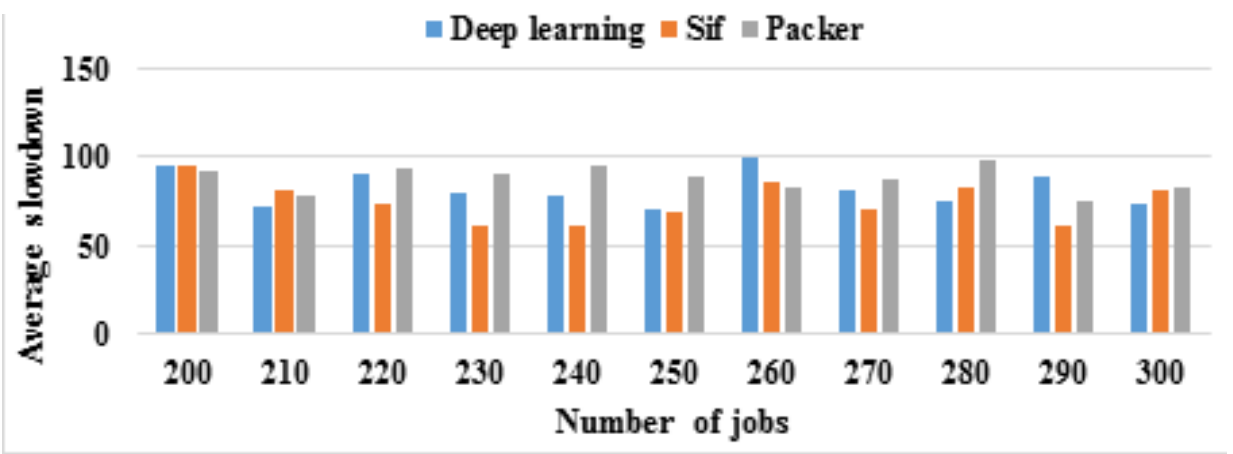

higher than that of the native deployment. It can be seen that the one-click deployment implemented in this article can speed up the deployment of the big data experimental environment and increase the success rate of deployment.

The job distribution of multiple virtual machines is shown in Figure 4. As can be seen from the figure, the number of jobs completed by each node basically meets the idea of load balancing, and there is no overload or excessive idleness of a node. When the probabilities of short jobs and small jobs decrease, the proportion of long jobs and jobs with large resources in the system increases, and the natural waiting situation also increases. Therefore, the downdown index of each algorithm tends to increase.

\subsection{Performance Test Results of Different Architectures}

The visualization result is shown in Figure 5. By analyzing the reasons, we can see that the training samples are appropriately divided and assigned to each Mapper to continue training. The smaller sample size makes the convergence speed on each Mapper training network faster, and even makes the whole training process faster. But when the sample segmentation cannot effectively cover the overall sample, a lot of cyclic training is needed. A total of three sets of sample data of different sizes were

\section{Figure 5. Visualization results}
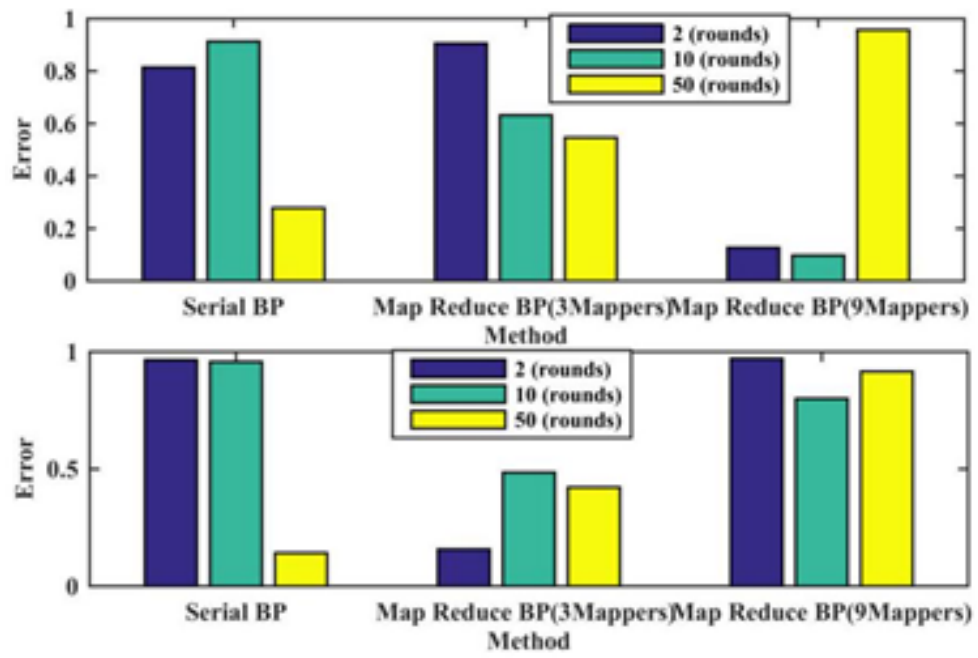
Figure 6. The relationship between MRBP algorithm's processing efficiency and data nodes

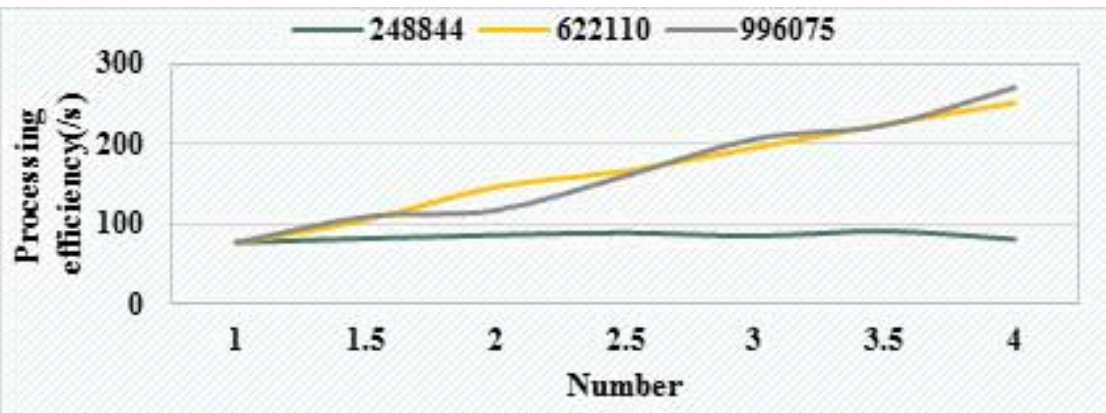

Table 3. Local drawing acceleration

\begin{tabular}{|l|l|l|l|l|}
\hline Number of nodes & Data1 & Data2 & Data3 & Data4 \\
\hline Single node & 1 & 1 & 1 & 1 \\
\hline Dual node & 1.227 & 1.790 & 1.792 & 1.753 \\
\hline Four node & 1.439 & 2.983 & 3.281 & 3.216 \\
\hline
\end{tabular}

obtained in the experiment: their capacities were $7.12 \mathrm{M}, 17.8 \mathrm{M}$, and $28.5 \mathrm{M}$, respectively. However, only the file size of sample data, such as $28.5 \mathrm{M}$, cannot accurately express the meaning of big data. Since the experiment is to train and classify all samples, the complexity of the calculation can be reflected by the number of samples. Because each sample is composed of numbers and belongs to the document file, the file capacity of the original sample data set is not large.

The relationship between MRBP algorithm's processing efficiency and data nodes is shown in Figure 6. When the number of samples is 248844 , the processing efficiency of the cluster does not become greater as the number of data nodes increases. That's because the file size is smaller than the set block. However, the cluster only uses one block to store files. According to the principle of proximity when processing data in Map-Reduce, the cluster actually only uses one node to train the data, so the processing time is almost not changing. From these tables and figures, it can be seen that the processing efficiency becomes greater as the number of samples increases, which also shows the advantages of cloud computing clusters in big data processing. The larger the data, the more obvious the advantage.

The local acceleration is shown in Table 3. The local rendering acceleration efficiency is shown in Table 4. It can be found from the table that the computing performance of double node is about 1.5-2 times that of single node; the computing performance of four node is about 3-3.5 times that of single node. Parallel rendering can greatly improve the rendering efficiency, and the more nodes, the greater the performance improvement. Looking at the graph of local rendering acceleration efficiency, we can find that the rendering acceleration efficiency of four nodes is slightly lower than that of two nodes, which indicates that when there are more nodes, the ratio of improving efficiency will

Table 4. Local drawing acceleration efficiency

\begin{tabular}{|l|l|l|l|l|}
\hline Number of nodes & Data1 & Data2 & Data3 & Data4 \\
\hline Single node & 1 & 1 & 1 & 1 \\
\hline Dual node & 0.614 & 0.895 & 0.896 & 0.877 \\
\hline Four node & 0.359 & 0.746 & 0.820 & 0.804 \\
\hline
\end{tabular}




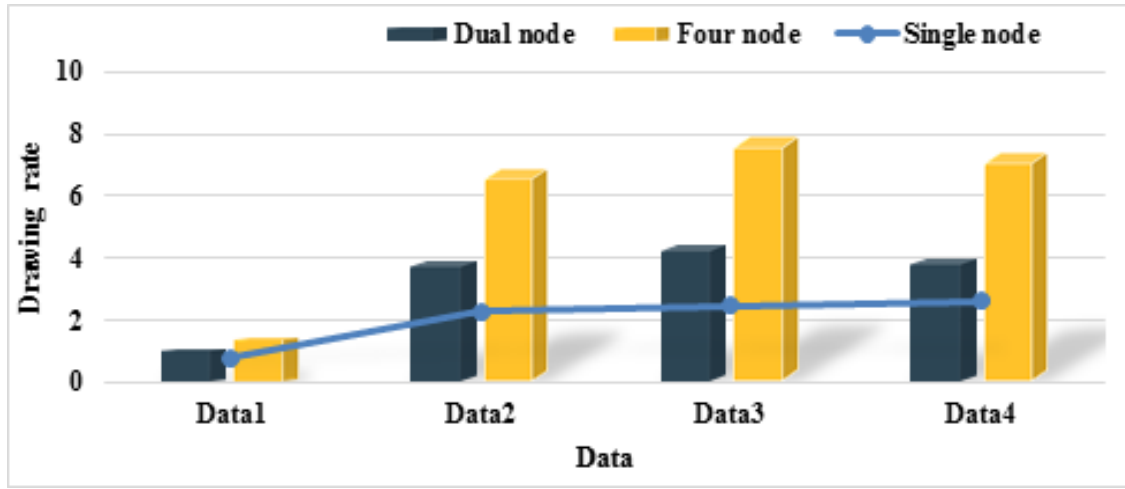

decrease. At the same time, from the acceleration and acceleration efficiency of local rendering, we can see that the efficiency of rendering datal is very low, which proves that when the amount of data is very small, the efficiency of rendering can not be greatly improved by using parallel mode, and the time of parallel processing small data can not be significantly reduced. And although more nodes will slow down the growth of performance, the overall computing performance is greatly improved.

\subsection{Results of the Total Digital Economy Estimation}

The local cluster drawing rate is shown in Figure 7. Comparing the rendering of different nodes, we can see that when drawing the same data, the rendering speed of multiple nodes is significantly faster than that of single node. However, from the rendering results of data1, it can be seen that when the amount of data is too small, cluster rendering can not improve too much speed, and the overall rendering speed is slow. However, when the amount of data increases, the rendering speed of cluster also increases greatly, and finally stabilized at a very high value. With the change of time, the system performance tends to be stable, and the throughput is slightly higher than that of LTBM. This is mainly because the LTBM method can simulate the particles in particle swarm optimization algorithm. In the process of searching for the optimal solution, it takes the global optimal position vector obtained by each iteration as the leader to quickly find the optimal virtual network mapping scheme in multi domain, which is conducive to searching for the appropriate task processing nodes for the corresponding tasks. This effectively ensures the resource utilization of the whole multi domain environment to a certain extent.

The prediction results and errors of the optimal combination prediction model based on BP neural network are shown in Table 5. The prediction error of the first mock exam is larger than that

Table 5. Forecast results and errors of the optimal combination forecasting model based on BP neural network

\begin{tabular}{|l|l|l|l|}
\hline Years & Actual mileage $(\mathbf{k m})$ & Neural network combination prediction $(\mathbf{k m})$ & Relative error(\%) \\
\hline 1990 & 26468 & 26457.84 & -0.384 \\
\hline 1991 & 27110 & 27098.75 & -0.415 \\
\hline 1992 & 27192 & 27212.88 & 0.768 \\
\hline 1993 & 28374 & 28341.20 & -1.156 \\
\hline 1994 & 29581 & 29618.48 & 1.267 \\
\hline 1995 & 31321 & 31300.89 & -0.642 \\
\hline
\end{tabular}


Table 6. Cointegration test results

\begin{tabular}{|l|l|l|l|l|}
\hline Null hypothesis & Characteristic root & Trace statistics & 5\% threshold & Prob \\
\hline None* & 0.733802 & 94.50938 & 69.81889 & 0.0002 \\
\hline At most 1* & 0.685369 & 58.77451 & 47.85613 & 0.0034 \\
\hline At most 2 & 0.459813 & 27.5529 & 29.79707 & 0.0888 \\
\hline At most 3 & 0.249659 & 10.92523 & 15.49471 & 0.2161 \\
\hline At most 4 & 0.11078 & 3.17008 & 3.841466 & 0.075 \\
\hline
\end{tabular}

of the single model, and the relative error is greater than $10 \%$ in a particular year. The combination forecasting model based on neural network has higher accuracy, better stability and error within $1 \%$. The individual is within $2 \%$, which shows that the whole is better than any three single prediction models.

The results of co integration test are shown in Table 6. From the co integration results, if the original hypothesis is rejected at a significant level of $5 \%$, there is a co integration relationship between the variables, that is, there is at least a long-term equilibrium between the respective variables and the dependent variables. The change of RMB exchange rate has the second influence on the stock of RMB in circulation abroad, that is, the stability of RMB value and the appreciation of RMB, which is an important reason why foreign countries are more willing to hold RMB and settle in RMB. Every $1 \%$ increase in the comprehensive score of digital economy development in the field of manufacturing will increase the global value chain division status index by $0.148 \%$. The development of digital economy will improve the production process of manufacturing industry, reduce its search cost and exchange cost in international trade, and indirectly enhance the creative effect of trade.

The proportion of China's digital economy scale in GDP is shown in Figure 8. It can be seen from the figure that the scale of the digital economy has increased from 250 billion yuan in 1996 to 27.17 trillion yuan in 2017, and its proportion in GDP has increased from less than 5\% in 1996 to $32.9 \%$ in 2017, an increase of nearly $30 \%$. From 1996 to 2005, the proportion of digital economy in GDP began to start. From 2005 to 2008, the growth was relatively slow. Since 2008, the proportion

Figure 8. The scale of my country's digital economy as a percentage of GDP

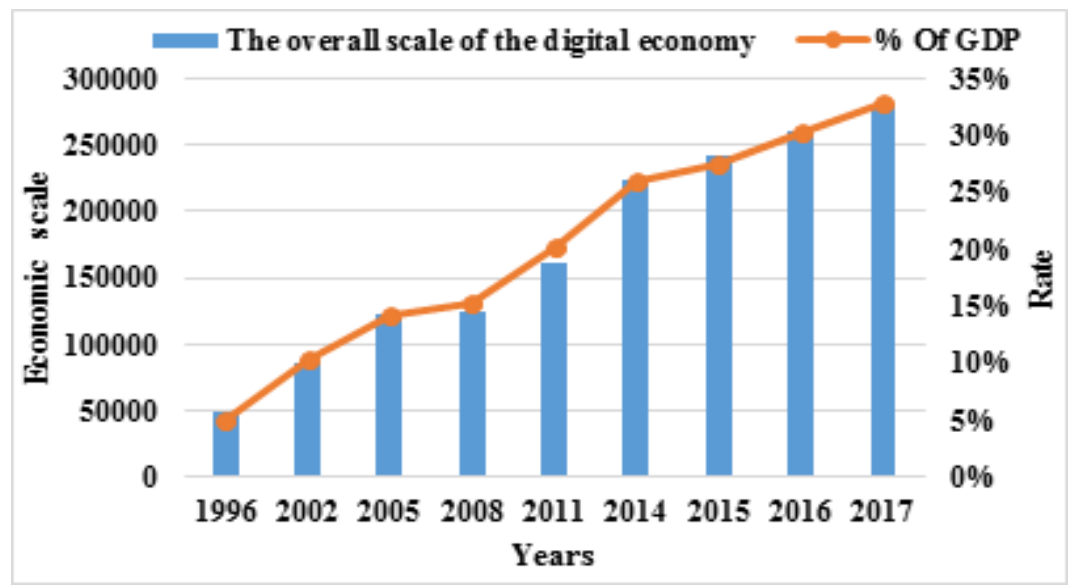


of digital economy in the overall economy has increased rapidly, reaching $32.9 \%$ by the end of 2017 . The basic part of digital economy is the leading force of digital development, the integration part is the main force of digital development, and the future national competitiveness is reflected in the integration part of digital economy.

\section{CONCLUSION}

This paper aims at efficient parallel processing of big data in cloud computing, and proposes a heuristic multi task deployment method LB-BC for long-term load balancing optimization of big data cloud platform in time dimension. In the long-term operation process of big data cloud platform, PS-ABC cooperates with dynamic load balancing migration of virtual machine to reduce the total incremental energy consumption of cloud platform, better protect the performance of virtual machine operation and migration, and promote the efficiency of multi task parallel computing for big data processing under cloud computing. When the short-term cross-border capital flows into China on a large scale, there will be obvious appreciation pressure on RMB, which makes the medium and long-term crossborder capital follow up one after another, in order to obtain RMB premium income. Moreover, large-scale cross-border capital inflow will push up inflation, create a large number of bubbles in the stock market and property market, shake the stability of the financial system, and affect the long-term development of the national economy.

\section{ACKNOWLEDGMENT}

This work was supported by China's National Social Science Research Grant project: Statistical Research on the Law of International Linkage of China's Business Cycle [16BTJ025](Janzhou Teng),Research on the Construction Path and Evaluation Mechanism of College Double Innovation Education Ecosystem, Key Research Projects of Higher Education in Jilin Province [JGJX2020C108]. (Yanmei Zhao),Research on high quality development of manufacturing industry in Jilin Province,Key projects of Jilin Province Social Science Foundation Projects in 2021(Binbin Zhu).

This work was supported by 2020 Shanghai Philosophy and Social Science Youth Project: Shanghai's producer service industry global value chain supply chain status assessment and promotion optimization research [2020EJB013]. (Yixin Zhou) 


\section{REFERENCES}

Albarqouni, S., Baur, C., Achilles, F., Belagiannis, V., Demirci, S., \& Navab, N. (2016). AggNet: Deep Learning from Crowds for Mitosis Detection in Breast Cancer Histology Images. IEEE Transactions on Medical Imaging, 35(5), 1313-1321. doi:10.1109/TMI.2016.2528120 PMID:26891484

Asaoka, R., Murata, H., Iwase, A., \& Araie, M. (2016). Detecting Preperimetric Glaucoma with Standard Automated Perimetry Using a Deep Learning Classifier. Ophthalmology, 123(9), 1974-1980. doi:10.1016/j. ophtha.2016.05.029 PMID:27395766

Biswas, S., Devi, D., \& Chakraborty, M. (2018). A Hybrid Case Based Reasoning Model for Classification in Internet of Things (Iot) Environment. Journal of Organizational and End User Computing, 30(4), 104-122. doi:10.4018/JOEUC.2018100107

Cao, B., Zhao, J., Lv, Z., Gu, Y., Yang, P., \& Halgamuge, S. K. (2020). Multiobjective Evolution of Fuzzy Rough Neural Network via Distributed Parallelism for Stock Prediction. IEEE Transactions on Fuzzy Systems, 28(5), 939-952. doi:10.1109/TFUZZ.2020.2972207

Charalampous, K., \& Gasteratos, A. (2016). On-line deep learning method for action recognition. Pattern Analysis \& Applications, 19(2), 337-354. doi:10.1007/s10044-014-0404-8

Chen, H., Fan, D., Huang, J., Huang, W., Zhang, G., \& Huang, L. (2020). Finite Element Analysis Model on Ultrasonic Phased Array Technique for Material Defect Time of Flight Diffraction Detection. Science of Advanced Materials, 12(5), 665-675. doi:10.1166/sam.2020.3689

Chen, Y., Lin, Z., Zhao, X., Wang, G., \& Gu, Y. (2017). Deep Learning-Based Classification of Hyperspectral Data. IEEE Journal of Selected Topics in Applied Earth Observations and Remote Sensing, 7(6), 2094-2107. doi:10.1109/JSTARS.2014.2329330

Edara, D. C., Sistla, V., \& Kolli, V. K. K. (2020). Health App Recommendation System using Ensemble Multimodel Deep Learning. Journal of Engineering Science and Technology Review, 13(5), 7-19. doi:10.25103/ jestr.135.03

Feng, Q., Li, Y., Wang, N., Hao, Y., Chang, J., Wang, Z., \& Wang, L. (2020). A Biomimetic Nanogenerator of Reactive Nitrogen Species Based on Battlefield Transfer Strategy for Enhanced Immunotherapy. Small (Weinheim an der Bergstrasse, Germany), e2002138. 10.1002/smll.202002138

Ghesu, F. C., Krubasik, E., Georgescu, B., Singh, V., Zheng, Y., Hornegger, J., \& Comaniciu, D. (2016). Marginal Space Deep Learning: Efficient Architecture for Volumetric Image Parsing. IEEE Transactions on Medical Imaging, 35(5), 1217-1228. doi:10.1109/TMI.2016.2538802 PMID:27046846

Hao, X., Zhang, G., \& Ma, S. (2016). Deep Learning. International Journal of Semantic Computing, 10(03), 417-439. doi:10.1142/S1793351X16500045 PMID:28113886

He, H., Wen, C. K., Jin, S., \& Li, G. Y. (2018). Deep Learning-based Channel Estimation for Beamspace mmWave Massive MIMO Systems. IEEE Wireless Communications Letters, 7(5), 852-855. doi:10.1109/ LWC.2018.2832128

Hosseini-Asl, E., Zurada, J. M., \& Nasraoui, O. (2016). Deep Learning of Part-Based Representation of Data Using Sparse Autoencoders with Nonnegativity Constraints. IEEE Transactions on Neural Networks and Learning Systems, 27(12), 2486-2498. doi:10.1109/TNNLS.2015.2479223 PMID:26529786

Kallenberg, M., Petersen, K., Nielsen, M., Ng, A. Y., Diao, P., Igel, C., Vachon, C. M., Holland, K., Winkel, R. R., Karssemeijer, N., \& Lillholm, M. (2016). Unsupervised Deep Learning Applied to Breast Density Segmentation and Mammographic Risk Scoring. IEEE Transactions on Medical Imaging, 35(5), 1322-1331. doi:10.1109/ TMI.2016.2532122 PMID:26915120

Kitouni, I., Benmerzoug, D., \& Lezzar, F. (2018). Smart Agricultural Enterprise System Based on Integration of Internet of Things and Agent Technology. Journal of Organizational and End User Computing, 30(4), 64-82.

Koesdwiady, A., Soua, R., \& Karray, F. (2016). Improving Traffic Flow Prediction with Weather Information in Connected Cars: A Deep Learning Approach. IEEE Transactions on Vehicular Technology, 65(12), 9508-9517. doi:10.1109/TVT.2016.2585575 
Li, W., Fu, H., Yu, L., Gong, P., Feng, D., Li, C., \& Clinton, N. (2016). Stacked Autoencoder-based deep learning for remote-sensing image classification: A case study of African land-cover mapping. International Journal of Remote Sensing, 37(23-24), 5632-5646. doi:10.1080/01431161.2016.1246775

Li, X., He, J., Hou, B., \& Zhang, P. (2018). Exploring the Innovation Modes and Evolution of the Cloud-Based Service Using the Activity Theory on the Basis of Big Data. Cluster Computing, 21(1), 907-922. doi:10.1007/ s10586-017-0951-z

Long, M., Wang, J., Cao, Y., Sun, J., \& Yu, P. S. (2016). Deep Learning of Transferable Representation for Scalable Domain Adaptation. IEEE Transactions on Knowledge and Data Engineering, 28(8), 2027-2040. doi:10.1109/TKDE.2016.2554549

Lv, Z., Hu, B., \& Lv, H. (2020). Infrastructure Monitoring and Operation for Smart Cities Based on IoT System. IEEE Transactions on Industrial Informatics, 16(3), 1957-1962. doi:10.1109/TII.2019.2913535

Lv, Z., \& Kumar, N. (2020). Software defined solutions for sensors in 6G/IoE. Computer Communications, l(153), 42-47. doi:10.1016/j.comcom.2020.01.060

Lv, Z., Liang, Q., Li, J. H., \& Song, H. (2020). Deep Learning Enabled Security Issues in the Internet of Things. IEEE Internet of Things Journal. Advance online publication. doi:10.1109/JIOT.2020.3007130

Mahmud, M., Kaiser, M. S., Hussain, A., \& Vassanelli, S. (2017). Applications of Deep Learning and Reinforcement Learning to Biological Data. IEEE Transactions on Neural Networks and Learning Systems, 29(6), 2063-2079. doi:10.1109/TNNLS.2018.2790388 PMID:29771663

Marmanis, D., Datcu, M., Esch, T., \& Stilla, U. (2016). Deep Learning Earth Observation Classification Using ImageNet Pretrained Networks. IEEE Geoscience and Remote Sensing Letters, 13(1), 105-109. doi:10.1109/ LGRS.2015.2499239

Nguyen, A., Yosinski, J., \& Clune, J. (2016). Understanding Innovation Engines: Automated Creativity and Improved Stochastic Optimization via Deep Learning. Evolutionary Computation, 24(3), 545-572. doi:10.1162/ EVCO_a_00189 PMID:27367139

Shankar, K., \& Elhoseny, M. (2019). Trust Based Cluster Head Election of Secure Message Transmission in MANET Using Multi Secure Protocol with TDES. Journal of Universal Computer Science, 25(10), 1221-1239.

Wang, C., Gong, L., \& Yu, Q. (2017). DLAU: A Scalable Deep Learning Accelerator Unit on FPGA. IEEE Transactions on Computer-Aided Design of Integrated Circuits and Systems, 36(3), 513-517.

Wang, J., Zhang, X., Gao, Q., Yue, H., \& Wang, H. (2017). Device-Free Wireless Localization and Activity Recognition: A Deep Learning Approach. IEEE Transactions on Vehicular Technology, 66(7), 6258-6267. doi:10.1109/TVT.2016.2635161

Wang, W., Zhang, M., Chen, G., Jagadish, H. V., Ooi, B. C., \& Tan, K.-L. (2016). Database Meets Deep Learning: Challenges and Opportunities. SIGMOD Record, 45(2), 17-22. doi:10.1145/3003665.3003669

Wang, X., Gao, L., Mao, S., \& Pandey, S. (2016). CSI-based Fingerprinting for Indoor Localization: A Deep Learning Approach. IEEE Transactions on Vehicular Technology, 66(1), 763-776. doi:10.1109/ TVT.2016.2545523

Wen, J., Yang, J., Jiang, B., Song, H., \& Wang, H. (2021). Big Data Driven Marine Environment Information Forecasting: A Time Series Prediction Network. IEEE Transactions on Fuzzy Systems, 29(1), 4-18. doi:10.1109/ TFUZZ.2020.3012393

Yan, Z., Zhan, Y., Peng, Z., Liao, S., Shinagawa, Y., Zhang, S., Metaxas, D. N., \& Zhou, X. S. (2016). MultiInstance Deep Learning: Discover Discriminative Local Anatomies for Bodypart Recognition. IEEE Transactions on Medical Imaging, 35(5), 1332-1343. doi:10.1109/TMI.2016.2524985 PMID:26863652

Yang, J., Wen, J., Jiang, B., \& Wang, H. (2020). Blockchain-based Sharing and Tamper-proof Framework of Big Data Networking. IEEE Network, 34(4), 62-67. doi:10.1109/MNET.011.1900374

Yeh, J. Y., \& Chen, C. H. (2020, July 16). A Machine Learning Approach to Predict the Success of Crowdfunding Fintech Project. Journal of Enterprise Information Management. Advance online publication. doi:10.1108/ JEIM-01-2019-0017 
Zeng, J., Tan, S., Li, B., \& Huang, J. (2016). Large-scale JPEG steganalysis using hybrid deep-learning framework. IEEE Transactions on Information Forensics and Security, 13(5), 1200-1214. doi:10.1109/TIFS.2017.2779446

Zhang, L., Tan, J., Han, D., \& Zhu, H. (2017). From machine learning to deep learning: Progress in machine intelligence for rational drug discovery. Drug Discovery Today, 22(11), 1680-1685. doi:10.1016/j. drudis.2017.08.010 PMID:28881183

Zhang, Y., He, Q., Xiang, Y., Zhang, L. Y., Liu, B., Chen, J., \& Xie, Y. (2017). Low-cost and confidentialitypreserving data acquisition for internet of multimedia things. IEEE Internet of Things Journal, 5(5), 3442-3451. doi:10.1109/JIOT.2017.2781737

Zhao, B., Feng, J., Wu, X., \& Yan, S. (2017). A survey on deep learning-based fine-grained object classification and semantic segmentation. International Journal of Automation and Computing, 14(02), 119-135. doi:10.1007/ s11633-017-1053-3

Zhao, W., \& Du, S. (2016). Spectral-Spatial Feature Extraction for Hyperspectral Image Classification: A Dimension Reduction and Deep Learning Approach. IEEE Transactions on Geoscience and Remote Sensing, 54(8), 4544-4554. doi:10.1109/TGRS.2016.2543748

Zhu, X. X., Tuia, D., Mou, L., Xia, G.-S., Zhang, L., Xu, F., \& Fraundorfer, F. (2018). Deep Learning in Remote Sensing: A Comprehensive Review and List of Resources. IEEE Geoscience and Remote Sensing Magazine, 5(4), 8-36. doi:10.1109/MGRS.2017.2762307

Yanmei Zhao, born in Baiyin City, Gansu province, China, 1986. She achieved the Master's degree from Jilin University of Finance and Economics.Now she is Studying for PhD in World Economics at Northeast Normal University. She works in School of Economics Management, Changchun Finance College. Her research directions include the Digital Economy, International Trade and Digital economy calculation and application.

Yixin Zhou was born in Xi'an, Shaanxi, P.R. China, in 1986. He received the doctor's degree from Harbin University of Commerce, Heilongjiang. P.R. China. Now, he works in School of Business Economics, Shanghai Business School. His research interests include the application of big data analysis in business economy and international trade. 